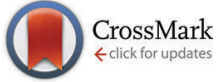

Cite this: Phys. Chem. Chem. Phys., 2016, 18, 6946

Received 2nd December 2015, Accepted 24th January 2016

DOI: $10.1039 / c 5 c p 07436 c$

www.rsc.org/pccp

\section{Evidence for electrohydrodynamic convection as a source of spontaneous self-ordering in porous anodic alumina films $\dagger$}

\author{
Mikhail Pashchanka* and Jörg J. Schneider \\ A comparative study of self-ordering behaviour of anodic alumina films fabricated in a series of diluted \\ (down to $0.05 \mathrm{M}$ ) oxalic acid electrolytes allowed developing a relationship between the supporting \\ electrolyte concentration and self-ordering voltages for the formation of porous oxide materials. Besides \\ its practical importance, this work elucidates some fundamental principles of porous alumina formation, \\ e.g. it suggests that the cell patterning arises from the electrohydrodynamic (EHD) convection process \\ rather than the interfacial tension gradients near the anode surface (Marangoni-type instability).
}

\section{Introduction}

A considerable part of contemporary research in nanotechnology is linked to self-organized materials, such as porous anodic aluminium oxide (PAOX) layers. ${ }^{1}$ For instance, porous alumina membranes can be used in nanofluidic systems, ${ }^{2-4}$ for the template synthesis of rod-like nanostructures, ${ }^{5}$ or even as nanoreactors, which can regulate the degree of polymerization by the spatial confinement effect. $^{6}$ For all these applications, the facile adjustment of both the structural parameters and the nonstoichiometric chemical composition of PAOX would be highly desirable. This could be achieved by increasing the number of suitable electrolytes for the optimized aluminium anodization conditions. However, a large share of well-organized mesoporous alumina films is still prepared for further laboratory tests via methods experimentally invented by Masuda et al. in the middle of 1990's (potentiostatic anodic oxidation of aluminium at a moderate voltage, and in most cases in a fairly concentrated single-solute aqueous electrolyte). ${ }^{7,8}$ It is commonly accepted that only voltages and concentrations from a narrow 'process window' lead to spontaneous self-organisation of a stable highly ordered nanoporous structure. ${ }^{9,10}$ These process windows are believed to be individual for every known electrolyte type, and are called the 'self-ordering regimes' ${ }^{11}$ Exploration of the spontaneous self-organisation in PAOX was initially motivated by numerous advantages of this approach over nanolithographic or nanoindentation texturing methods, especially in terms of

Fachbereich Chemie, Eduard-Zintl-Institut, Fachgebiet Anorganische Chemie, Technische Universität Darmstadt, Alarich-Weiss-Straße 12, 64287, Darmstadt, Germany. E-mail: mikhail.pashchanka@gmail.com

$\dagger$ Electronic supplementary information (ESI) available: A more detailed set of microscopic analysis data for PAOX samples obtained at all studied electrolyte concentrations and all tested voltages in sequence. See DOI: 10.1039/c5cp07436c simplicity and manufacturing cost (this was also emphasized in the early work of Masuda). However, in an attempt to alter the pore size and shape, many alternative approaches based on artificial pre-patterning of pore nucleation sites on aluminium substrates have been developed. ${ }^{12-14}$ At the same time, different factors influencing the growth behaviour of self-organized nanoporous arrays, such as electrolyte type ${ }^{15}$ concentration, ${ }^{16} \mathrm{pH},{ }^{17}$ voltage ${ }^{18}$ and temperature ${ }^{16,19}$ have been intensively studied. Recently, we have proposed an empirical relationship between these parameters (some of them are included indirectly): ${ }^{20}$

$$
P=\frac{q_{\mathrm{av}} \Delta U}{\eta \sigma}
$$

where $P$ is the criterion for the appearance of PAOX cell structure (the critical value was experimentally estimated for the most widely used electrolytes to be close to 0.057$), q_{\mathrm{av}}$ is the average charge of supporting electrolyte anions (modulo, $q_{\mathrm{av}}=10^{-\mathrm{pH}} / C, C$ is the known acid concentration), $\Delta U$ is the applied voltage, $\eta$ is the dynamic viscosity, and $\sigma$ is the specific electric conductivity. Many recent theoretical and experimental studies show that the hexagonal patterning in PAOX is of convective origin..$^{20-23}$ As the possible driving forces for nanoconvection, either the interfacial tension gradient near the anode or the interaction of the electric field with charge carriers is usually considered. Other models attempting to explain the hexagonal pore arrangement are also in partial agreement with the convective model; however, they do not completely validate the process. ${ }^{21,24}$ For instance, the study featuring the tensile stress distribution as a possible factor assisting the pore ordering ${ }^{24}$ demonstrated the diffusion of tracer elements in alumina from the pore base to the pore walls. This experimental observation fully agrees with the nano-convective mechanism and does not give rise to a severe contradiction between two approaches 
(it is worth mentioning though that the convection is usually defined $^{23}$ as a collective flow of particles in fluids and gases, but not in solids). However, the theoretical simulation of the viscous oxide flow in this work ${ }^{24}$ rests on the overly simplistic model of PAOX: a homogeneous $\mathrm{Al}_{2} \mathrm{O}_{3}$ continuum without electrolyte anion inclusion. This model neglects experimental observations such as the colloidal nature of the amorphous films, structural defects and flaws, bound water (up to $5.7 \mathrm{wt} \%$ ), and the electrolyte species content up to $9.84 \mathrm{wt} \% .^{25-27}$ It is well known that the presence of grain boundaries and structural defects can significantly change the mechanisms and velocity of ion diffusion (the so-called grain-boundary diffusion). Thus, the concept of colloidal self-organisation ${ }^{20,26}$ is more consistent with experimental facts. It is very likely that the hexagonal cell arrangement in PAOX arises similarly to the patterns observed upon establishing the electrohydrodynamic (EHD) convection in colloidal systems (in the case of PAOX, this can be an in situ formed suspension layer of mobile charged alumina particles). ${ }^{23}$ Another convincing experimental fact that counts in favour of the structure-forming role of convection is the periodic damped $I-t$ (electric current versus time) oscillation, which is typical for PAOX growth and anodic dissolution of some other metals where surfaces are etched in the form of hexagons due to a convective process. $^{23}$ For instance, the shapes of $I-t$ curves are almost identical for the aluminium anodization in a mixed electrolyte containing cerium salt ${ }^{28}$ and the potentiostatically controlled $\mathrm{Cu}\left|\mathrm{CuSO}_{4}\right| \mathrm{Cu}$ electrolytic cell where the electrochemical analogue of RayleighBénard convection takes place. ${ }^{29}$

In this study, we explore the self-ordering behaviour of PAOX layers formed at new low concentrations of oxalic acid electrolyte (decreased down to $0.05 \mathrm{~mol} \mathrm{~L}^{-1}$, which is six times lower than that conventionally used for the well-established mild anodization regimes ${ }^{7}$ ). We systematically change the electrolyte concentration and explore new process windows for these nontrivial electrolyte solutions. This will provide the experimental test of our earlier hypothesis that the convective currents responsible for the hexagonal pattern can occur even at very low electrolyte concentrations, approaching deionised water. ${ }^{22}$ However, a sufficient amount of electrolyte is needed to ensure acidic $\mathrm{pH}<4$, since only electrolytes in which alumina is slightly soluble are known to produce porous solid films. ${ }^{30}$ This specific condition is concerned with the chemistry of PAOX formation (stabilisation of the in situ formed colloidal alumina particles) rather than establishing nano-convection, which is responsible for self-organisation. ${ }^{20}$ In addition, we test the interval of applicability of eqn (1), as well as the validity of the earlier found critical value of the criterion $P$, for different solutions. The clarified relation of concentration and self-ordering voltages will facilitate the design of further electrochemical experiments with free choice of electrolyte composition. Our experiments can improve our knowledge of the PAOX formation mechanism, particularly, of the possible driving force for nano-convection.

\section{Results and discussion}

\section{Theoretical prediction of self-ordering voltages for new electrolyte concentrations using the earlier found value of $\boldsymbol{P}$}

In order to determine the new self-ordering regimes, it is helpful to start with the measurement of macroscopic parameters of diluted electrolyte solutions and estimation of the new selfordering voltages using eqn (1), and then refining these voltages experimentally. Before we begin the analysis of the electrolyte properties, one important detail must be mentioned regarding eqn (1) and the criterion $P$ from ref. 20. The critical number $P$ for honeycomb structure formation was found using the measured parameters of three well-known classical electrolytes for PAOX synthesis, i.e. 0.3 M oxalic acid, 0.3 M sulphuric acid, and $10 \mathrm{wt} \%$ phosphoric acid solutions. These compositions displayed $\mathrm{pH}$ close to 1.00 , and thus were measured in the so-called 'acidic error range', where a glass electrode shows noticeably overestimated $\mathrm{pH}$ values. Although these readings cannot very largely influence the calculated values of $q_{\text {av }}$ and $P$, we must bear in mind this general shortcoming of glass electrodes. Table 1 summarizes the measured properties of the diluted oxalic acid solutions, including the dynamic viscosities, specific electric conductivities, and $\mathrm{pH}$. As one can see, the average absolute anionic charge $\left|q_{\mathrm{av}}\right|$, which is derived from $\mathrm{pH}$ measurements, decreases with higher concentrations. This effect is entirely clear because the degree of dissociation is larger for diluted solutions. However, 0.3 M composition slightly deviates from the general dependence. This observation is very likely caused by the non-linear response of the glass $\mathrm{pH}$ electrode when approaching low $\mathrm{pH}$ values of about 1.00 (the above-mentioned instrumental 'acidic error'). Eqn (1) suggests voltages appropriate for the transition from non-porous to honeycomb alumina

Table 1 Measured parameters of $\mathrm{H}_{2} \mathrm{C}_{2} \mathrm{O}_{4}$ solutions at $0{ }^{\circ} \mathrm{C}$, calculated and experimentally refined voltages, observed pore diameters $d_{\mathrm{p}}$, and revised values of criterion $P$

\begin{tabular}{|c|c|c|c|c|c|c|c|c|c|}
\hline \multirow[b]{2}{*}{ Concentration (M) } & \multicolumn{4}{|c|}{$\begin{array}{l}\text { Measured macroscopic } \\
\text { solution parameters }\end{array}$} & \multirow{2}{*}{$\begin{array}{l}\text { Calculated } \\
\Delta U^{b}(\mathrm{~V})\end{array}$} & \multirow{2}{*}{$\begin{array}{l}\text { Initial } \\
d_{\mathrm{p}}(\mathrm{nm})\end{array}$} & \multirow{2}{*}{$\begin{array}{l}\text { Critical } \Delta U(\mathrm{~V}) \\
\text { refined by } \\
\text { experiment }^{c}\end{array}$} & \multirow{2}{*}{$\begin{array}{l}d_{\mathrm{p}}(\mathrm{nm}) \text { after } \\
\text { optimization }\end{array}$} & \multirow{2}{*}{$\begin{array}{l}\text { Specific } \\
\text { numerical } \\
\text { values of } F\end{array}$} \\
\hline & $\eta(\mathrm{cP})$ & $\sigma\left(\mathrm{mS} \mathrm{cm}^{-1}\right)$ & $\mathrm{pH}$ & $\left|q_{\text {av }}\right|$ & & & & & \\
\hline 0.2 & 1.88 & 40.5 & 1.54 & 0.144 & 30 & $18 \pm 2$ & 43 & $27 \pm 2$ & 0.081 \\
\hline 0.1 & 1.86 & 26.1 & 1.69 & 0.204 & 14 & $9 \pm 1$ & 44 & $24 \pm 2$ & 0.185 \\
\hline 0.05 & 1.81 & 16.1 & 1.80 & 0.317 & 5 & $6 \pm 1$ & 45 & $32 \pm 2$ & 0.490 \\
\hline
\end{tabular}

${ }^{a}$ Solution properties are taken from ref. 20, optimized $\Delta U$ and the corresponding $d_{\mathrm{p}}$ from ref. $7 .{ }^{b}$ Approximated to whole numbers. ${ }^{c}$ Determined by the optimal combination of morphological features such as the size of long-range ordered domains and the regular hexagonal shape of defectfree cells containing single pores. 
A

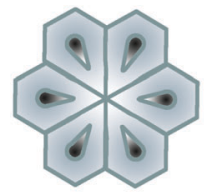

B
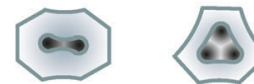

C
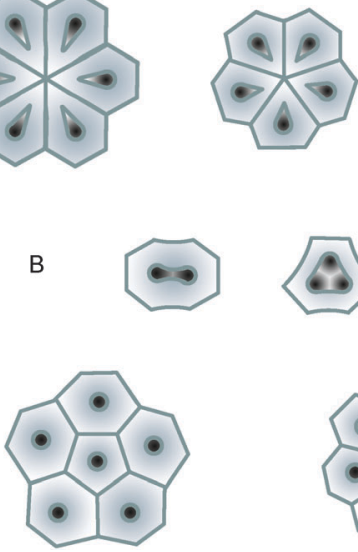

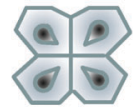

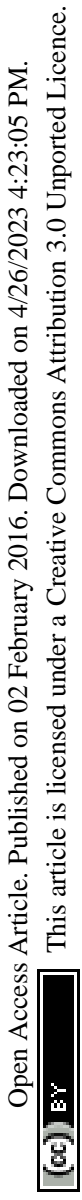

.

for all the oxalic acid concentrations under study; the specific electric conductivities are above the minimal required value of the self-assembly $\left(\sigma=4-5 \mathrm{mS} \mathrm{cm}^{-1}\right)$ in all cases as well. ${ }^{22}$ Thus, our calculations indicate that the formation of nanoporous alumina films is possible in all the selected electrolyte compositions. The theoretically predicted self-organization voltages from Table 1 are calculated for $P=0.057$, i.e. for the centre of

Scheme 1 Basic types of point defects in PAOX: (A) missing pores, surrounded by a different number of satellites; (B) multiple pores within one cell; (C) pores surrounded by 5 or 7 neighboring pores (rarely 8 ) instead of 6 . The five-fold and seven-fold coordinated pores are usually adjacent to each other to annihilate the strain. Besides the depicted defects, cells with six satellites and single pores within, but with strongly distorted geometry can also be mentioned as local imperfections.

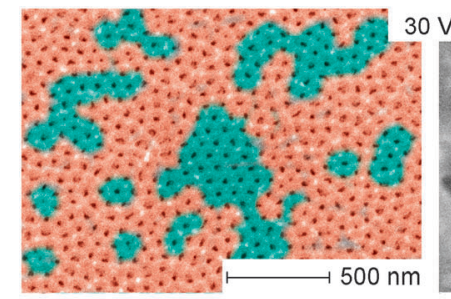

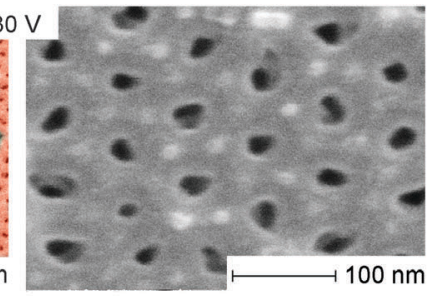
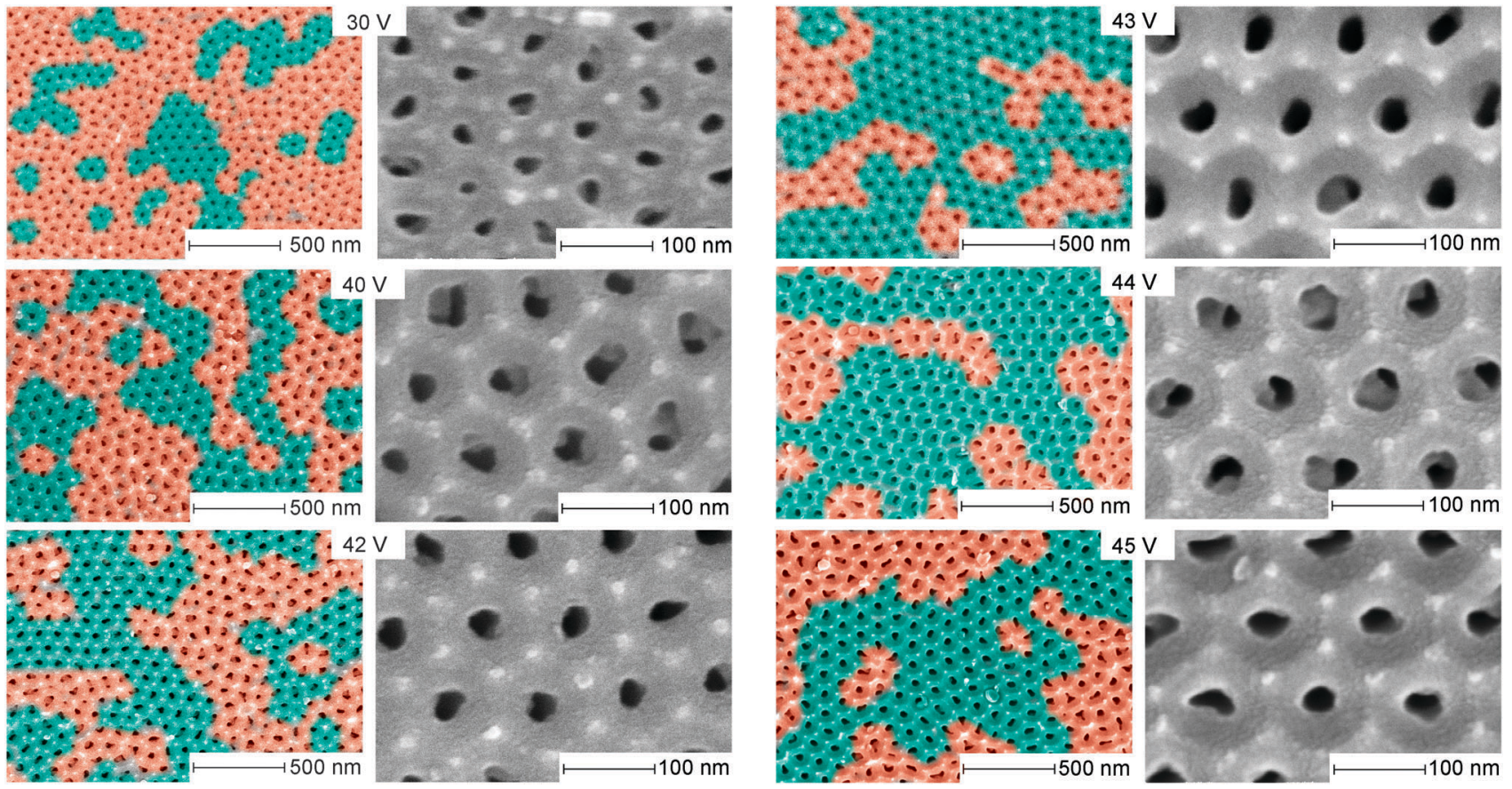

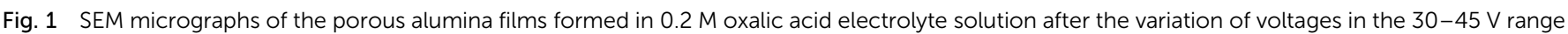

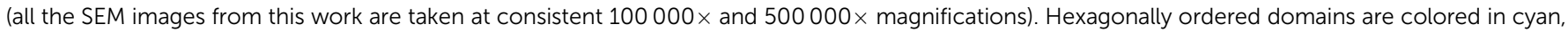

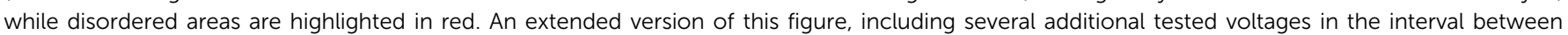
30 and $41 \mathrm{~V}$, as well as at $\Delta U$ exceeding $45 \mathrm{~V}$, is available in the ESI $\uparrow$ in Fig. S1. 
are still up to $2.5-3 \mu \mathrm{m}$ large, but double pores within a cell start to occur frequently. Such point defects often denote that the oxidation voltages exceed the optimal values. For instance, if anodization is conducted in $0.3 \mathrm{M}$ solution, but at an increased voltage of $60 \mathrm{~V}$, the majority of cells in the resulting PAOX layers will have double or triple pore openings (see Fig. S2, ESI $\dagger$ ). Thus, we can consider $42-43 \mathrm{~V}$ as the suitable critical voltages for $0.2 \mathrm{M}$ oxalic acid, above which the convection preferentially appears in the form of long-range ordered hexagonal cells, and 44-45 $\mathrm{V}$ as the upper limit of the optimal process window. Due to irregular shapes of hexagonally ordered domains, an accurate comparison of their sizes at different oxidation voltages is difficult. Thus, the found process window of $42-45 \mathrm{~V}$ has slightly uncertain limits because of the mainly qualitative assessment of the degree of ordering. It should also be noted that the PAOX layers formed in a $0.2 \mathrm{M}$ electrolyte solution demonstrated a higher stability of the hexagonal pattern than layers from more diluted compositions, which are discussed later on in this work, as well as higher tolerance to moderate voltage variations. So, even at $32 \mathrm{~V}$ (which is $10-11 \mathrm{~V}$ lower than the estimated critical $\Delta U$ ), the ordered domain size was still $0.2-0.8 \mu \mathrm{m}$. Of course, a smaller specific electric conductivity of $0.2 \mathrm{M}$ oxalic acid and weaker ionic currents during the electrolysis (in comparison with the commonly used $0.3 \mathrm{M}$ concentration) cannot provide the same degree of aluminium substrate pre-texturing (in the form of hexagonally ordered uniform concaves, underlying the alumina cells), and the remaining natural defects, such as pits, ridges and scratches, will bring distortions to the hexagonal layout. Although the experimentally refined critical voltage belongs to the initially specified confidence interval $30 \pm 13 \mathrm{~V}, P$ evidently needs recalculation for the new electrolyte concentration. If we accept $43 \mathrm{~V}$ as the new critical voltage, the value of $P$ is equal to 0.081 , which considerably exceeds $P=0.055$ for the classical $0.3 \mathrm{M}$ oxalic acid. ${ }^{20}$ As was established in our preceding paper, ${ }^{22}$ the earlier found value of criterion $P$ can be actually underestimated for weaker diluted organic acids, and very likely needs a correction for a better hexagonal pore arrangement. Taking into account the large mismatch between the previous and the current values of $P(47.3 \%)$, this result cannot be solely ascribed to the moderate instrumental error during $\mathrm{pH}$-measurements, and has another underlying reason. As we show later on in this work, the criterion $P$ appears to be indeed non-constant (systematically changed) for different supporting electrolyte concentrations.

\section{Experimental refinement of anodizing voltage in $0.1 \mathrm{M}$ oxalic acid}

According to prior calculations, the predicted self-ordering voltage for $0.1 \mathrm{M}$ anodizing electrolyte lies presumably within the interval $14 \pm 6 \mathrm{~V}$. Oxidation at $14 \mathrm{~V}$ resulted in an entirely disordered pore layout (see Fig. 2). No hexagonal structure was observed at the upper confidence limit as well (see the SEM images of the samples obtained at $19 \mathrm{~V}$ and $21 \mathrm{~V}$ in Fig. 2). The first sporadically ordered fragments occurred only at $25 \mathrm{~V}$ and had dimensions up to $0.4 \mu \mathrm{m}$. As the voltage increases, the regularity in the pore layout slightly improves. However, PAOX layers formed at practically all tested voltages showed high randomness in the pore arrangement, and the tendency to ordering was noticeably lower than during the experiments in $0.2 \mathrm{M}$ oxalic acid. The most stable and reproducible hexagonal patterns were obtained at the voltages of 43-44 V. This incipient hexagonal arrangement was fully lost already at the voltage of $45 \mathrm{~V}$. Moreover, the $45 \mathrm{~V}$ PAOX layers frequently demonstrated the same type of point imperfections as earlier discussed for the films formed in $0.2 \mathrm{M}$ solution at excess voltages, i.e. double pores within one cell (see the corresponding close-up SEM image in Fig. S3, ESI $\dagger$ ). Thus, the $44 \mathrm{~V}$ potential difference can also be considered as the upper limit of the narrow optimized process window. As in the case of $0.2 \mathrm{M}$ anodizing electrolyte, the experimentally refined self-ordering voltage for the $0.1 \mathrm{M}$ composition is beyond the theoretically predicted range, and the criterion $P$ certainly needs a correction. If we take $44 \mathrm{~V}$ as the critical voltage for the $0.1 \mathrm{M}$ electrolyte concentration, the new value of $P$ is equal to 0.185 , and thus exceeds both previous results for the more concentrated $0.2 \mathrm{M}$ and $0.3 \mathrm{M}$ compositions. The discrepancy between the predicted self-ordering voltages and the experimental critical $\Delta U$ for $0.1 \mathrm{M}$ oxalic acid is about $23-24 \mathrm{~V}$, and the deviation from the previously found value of the critical number $P$ is now $236 \%$. Now, it is beyond any doubt that the gap between the values calculated using eqn (1) and the empirical values results from reasons other than the measuring error during the $\mathrm{pH}$ determination. On the other hand, the mismatch between the predicted and experimentally optimized critical voltages and the criterion $P$ systematically increases, when approaching low electrolyte concentrations. This suggests that the critical parameters for the self-ordering can depend on the anodizing electrolyte concentration, and even have a functional correlation with it.

\section{Experimental refinement of anodizing voltage in $0.05 \mathrm{M}$ oxalic acid}

Calculations using $P$ from ref. 20 suggest that the self-ordering in the most diluted $0.05 \mathrm{M}$ electrolyte should occur at approximately $6 \pm 1 \mathrm{~V}$. The films obtained at the lowest test voltage of $5 \mathrm{~V}$ have a well-defined porous structure. However, instead of a cell pattern, they revealed a spongy morphology (Fig. 3). No hexagonal ordering could be observed even at voltages about 10-20 V higher than initially predicted by eqn (1). For the $0.05 \mathrm{M}$ anodizing electrolyte, an optimal combination of the regular hexagonal shape of individual cells and the large size of ordered domains (up to $1 \mu \mathrm{m}$ ) occurs at the voltage of $45 \mathrm{~V}$, which very likely should be considered as critical for this concentration. At $46 \mathrm{~V}$, the cells acquire even a clearer and sharper hexagonal form, but the size of ordered domains becomes noticeably smaller. At higher voltages, the hexagonal pattern disappears completely, and individual cells begin to suffer from defects such as double and triple pores (see the corresponding SEM images of the sample obtained at $48 \mathrm{~V}$ in Fig. S4 of the ESI $\dagger$ ). If we consider $45 \mathrm{~V}$ as the new critical voltage for $0.05 \mathrm{M}$ oxalic acid, the corrected new value of $P$ is 0.490 . Thus, it went up by a factor of 8.9 in comparison with the critical number for $0.3 \mathrm{M}$ oxalic acid electrolyte. It should be mentioned that the comparison of the samples obtained from $0.05 \mathrm{M}$ oxalic acid is fairly difficult because of the poor 

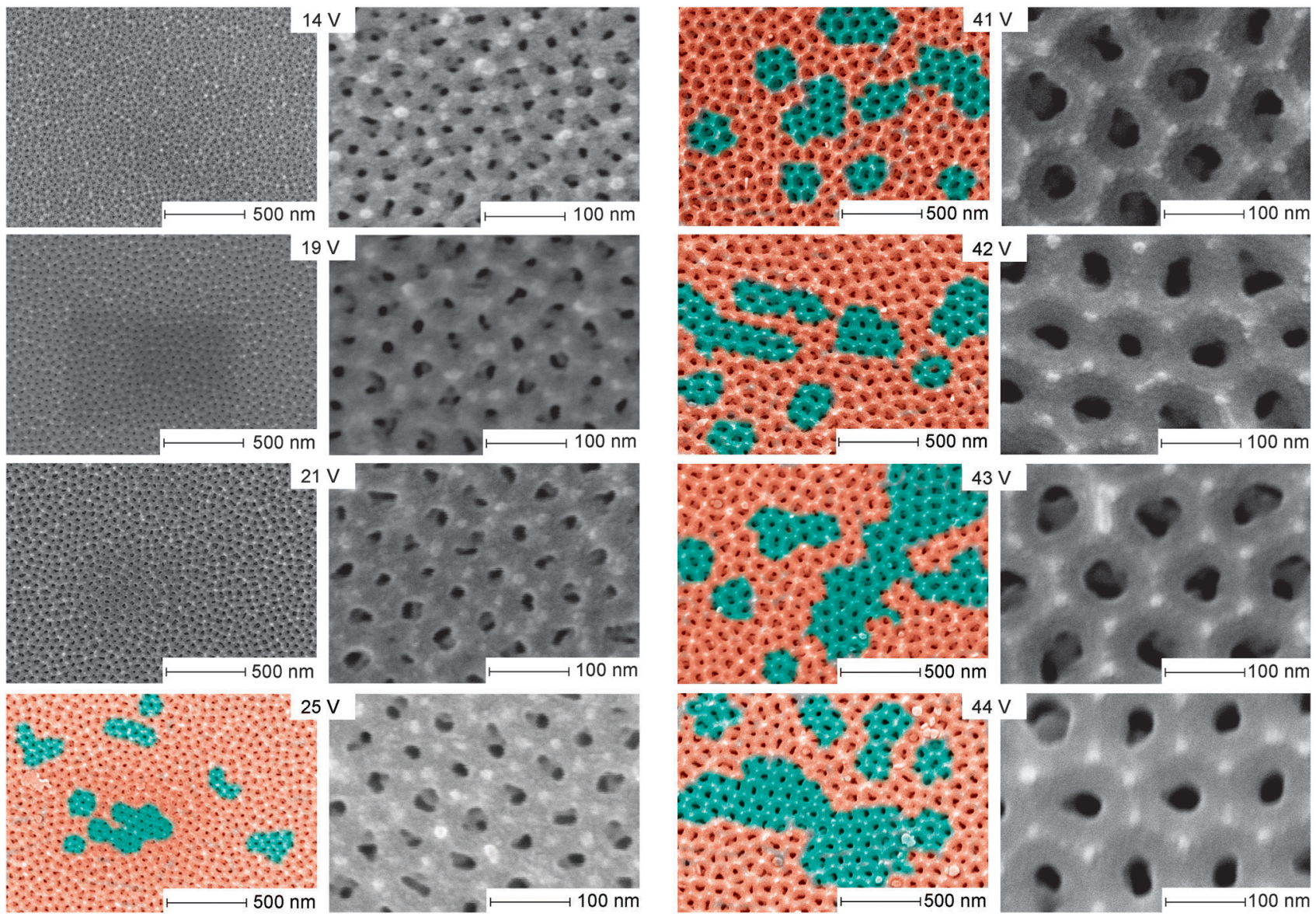

Fig. 2 SEM examination of the PAOX films obtained from $0.1 \mathrm{M}$ oxalic acid anodizing electrolyte at voltages within the range of 14-44 $\mathrm{V}$ (close-up images are taken at $500000 \times$ magnification, and the overall views at $100000 \times$ ). The extended version of this microscopic analysis with additional tested voltages is demonstrated in Fig. S3 of the ESI. $\dagger$ The color scheme showing ordered and disordered areas on the PAOX layers is identical to the one used in Fig. 1: ordered domains are highlighted with cyan, and disordered fields with red.

self-ordering in practically all cases. Very low supporting electrolyte concentration cannot provide sufficient current densities for the efficient structuring of the $\mathrm{Al}$ substrate in the form of hexagons, and for maintaining a reasonable reaction velocity for spontaneous rearrangement into a well-organized pattern. In the whole range of tested voltages, self-ordered domains were observed as sporadic structures in mainly disordered PAOX, and only somewhat intuitive selection based on qualitative assessment was possible. When determining the critical voltage for the most diluted solution, we should also pay attention to the general trend in the whole survey of all other tested concentrations.

\section{Theoretical analysis of the experimental data}

At first glance, there is a colossal mismatch between the theoretical calculations and the experimental results at low supporting electrolyte concentrations (see Fig. 4 for comparison), which may suggest that the empirical eqn (1) is invalid. However, we can easily explain this seeming difference if we assume that the honeycomb structure in PAOX is a product of the electrohydrodynamic (EHD) convection, which is driven by the interaction of the electric field $E$ with excess space charge density $q_{\mathrm{ex}}$ in the solution (volume electric forces). Here, it would be helpful to start with the comparative analysis of eqn (1) and another rigorously theoretically derived and experimentally confirmed expression for the electrical analogue of Rayleigh number $R a_{\mathrm{e}}$, which determines the critical conditions, above which the EHD convection sets in: $:^{23,31}$

$$
R a_{\mathrm{e}}=\frac{\varepsilon_{\mathrm{r}} \varepsilon_{0} \Delta U}{u_{\mathrm{s}} \eta}
$$

where $\varepsilon_{\mathrm{r}}$ is the relative permittivity of the fluid, $\varepsilon_{0}$ is the permittivity of vacuum, $u_{\mathrm{s}}$ is the ionic mobility of species $s$ (i.e. of the charge carriers, contributing to the Faraday current), and the rest of the parameters in the second part of eqn (2) entirely corresponds to the parameters of eqn (1). Now, if we look at the physical meaning of the ionic mobility $u_{\mathrm{s}}$, it can be defined as the electric conductivity at infinite dilution. Ionic mobility was initially considered for the description of the solution electric properties in eqn (1), but specific electric conductivity $\sigma$ was introduced instead because it is easily measurable and suitable for the routine laboratory prediction of new self-ordering regimes. ${ }^{20}$ The part of the numerator $\varepsilon_{\mathrm{r}} \varepsilon_{0}$ is linked to the excess space charge density carried by the liquid, as expressed by the Poisson equation:

$$
q_{\mathrm{ex}}=\varepsilon_{\mathrm{r}} \varepsilon_{0} \operatorname{div} E
$$



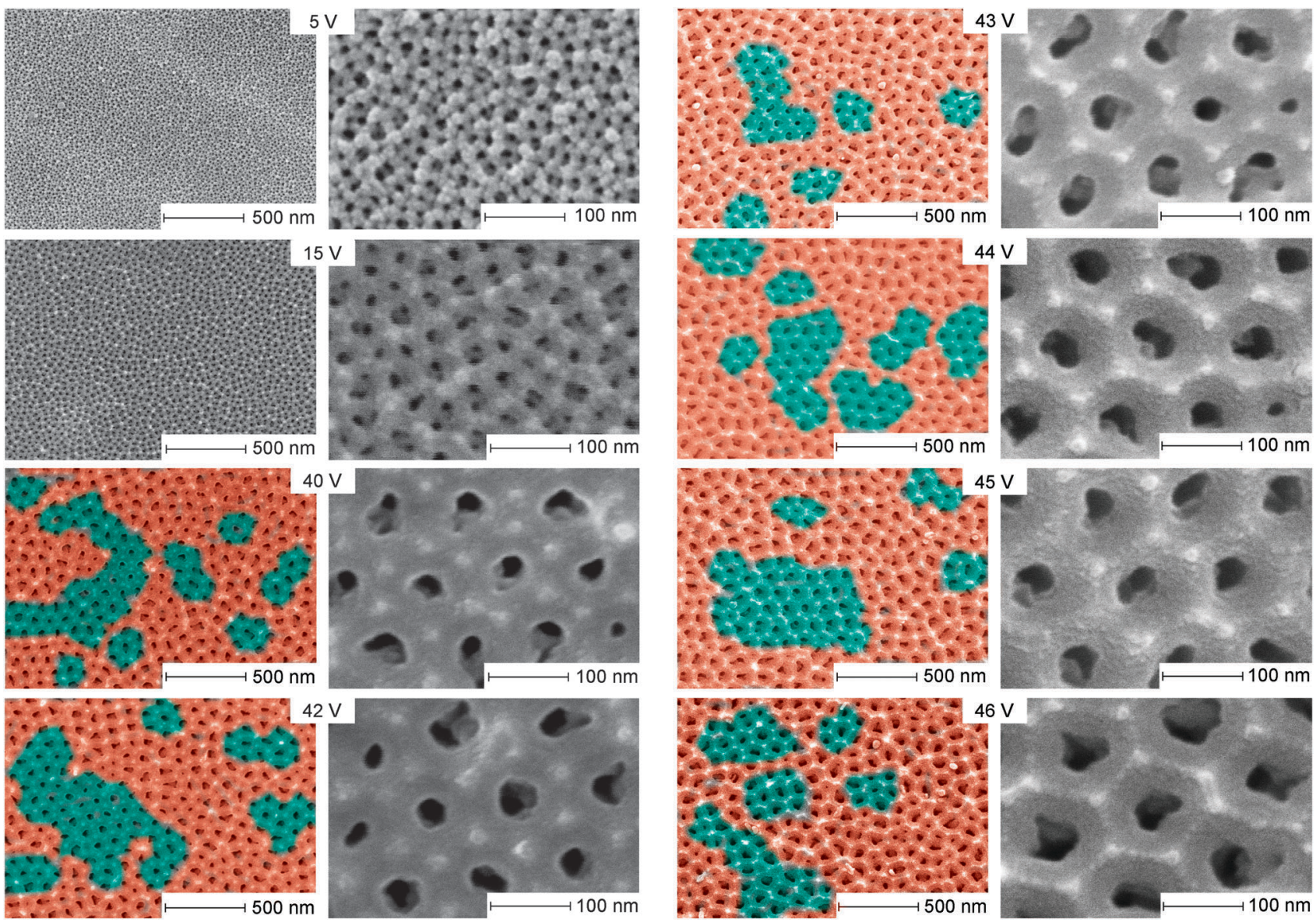

Fig. 3 SEM micrographs of the PAOX films formed in $0.05 \mathrm{M}$ oxalic acid electrolyte at different anodizing voltages. While the regular hexagonal shape of individual cells can be observed at a number of voltages above $40 \mathrm{~V}$, the size of ordered domains is relatively small at all tested voltages, and reaches the largest reproducible values at $45 \mathrm{~V}$. The tendency to self-ordering at the voltages of $40-42 \mathrm{~V}$ seems surprising because of the conflict with the general trend of critical voltages for other tested electrolyte concentrations. It is still not entirely clear, whether the self-ordering can improve repeatedly in the same solution, as the voltage increases.

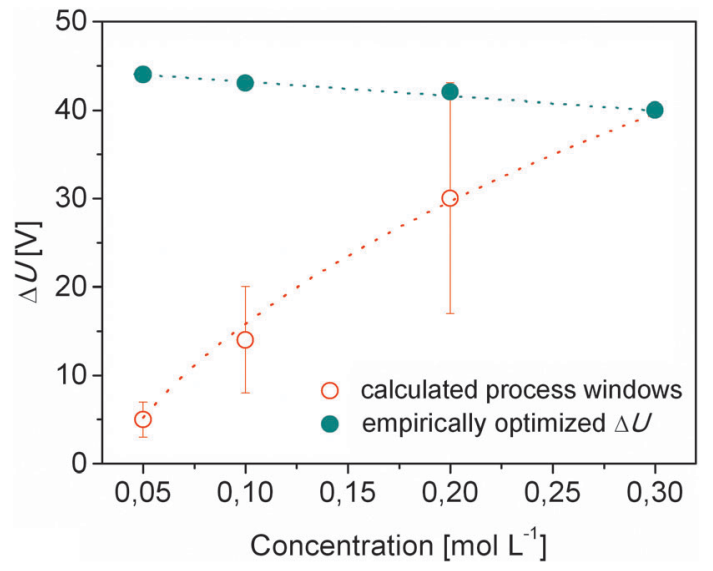

Fig. 4 Comparison of the calculated process windows using $P=0.057 \pm$ 0.024 for all tested concentrations, and experimentally optimized self-ordering voltages for aluminium anodization in 0.05-0.3 M oxalic acid electrolytes.

which has a close relation to the parameter $q_{\mathrm{av}}$ from eqn (1) ( $q_{\mathrm{av}}$ is determined as the ratio of the total negative charge of all anions in the solution to the supporting electrolyte concentration $C$ ).
Hence, although eqn (1) was found and confirmed only empirically, it appears to be written strikingly similar to the theoretically derived expression for the critical dimensionless number for the onset of the EHD convection.

For the appearance of the EHD convection in a nonconducting non-electroactive medium (i.e. in a purely physical system), a high voltage of the order of $10^{4} \mathrm{~V}$ is required. ${ }^{23}$ However, in the presence of even trace amounts of conducting species, the required voltages for the development of sufficient spatial charge and the onset of the EHD convection drop to a few volts. Moreover, the required critical voltage decreases further, if the unipolar charge injection (i.e. the injection of ions of the same sign from the side of only one electrode) is substituted by the bipolar charge injection (when charge carriers of both signs are injected into the solution from the opposite electrodes). For instance, the initially experimentally confirmed critical value of $R a_{\mathrm{e}}$ from eqn (2) appeared to be invalid for the systems with symmetrical bipolar charge injection. In such electrochemical systems, lower critical voltages were needed for the onset of the EHD convection, and the new critical value of $R a_{\mathrm{e}}$ was approximately two times smaller than the previously determined. 
This situation is totally identical to what we observe in our current investigation of the electrochemical system for the PAOX growth: as summarized in Table 1, the critical value of the criterion $P$ drops from 0.490 to 0.055 , as the supporting electrolyte concentration increases from $0.05 \mathrm{M}$ to $0.3 \mathrm{M}$ (also see Fig. 5 for graphic comparison). Here, however, the complexity of the electrochemical cell for PAOX formation, as well as the complexity of water as solvent, must be mentioned. While physical systems seem to be the perfect models for isolation and a separate study of different factors that can affect the onset of instability (in other words, one can start with the simplest plain non-conducting non-electroactive fluid, then add charge carriers in different proportions, then test unipolar, bipolar injections etc.), the electrochemical cell for PAOX synthesis always includes a tight indivisible combination of all parameters, with a very complex interplay between them. Firstly, although pure 'deionised' water has a high dielectric constant, strictly speaking, it is never completely ion-free because of the dissociation of water molecules. Secondly, water plays the roles of the fluid medium and the electro-active species at the same time, and the electrode potentials of the redox reactions with participation of water depend on $\mathrm{pH}$ (i.e. the concentration of supporting electrolyte). Finally, the opposite electrodes during aluminium anodization make only one kind of charge carrier injection possible, which results from the generation of protons at the anode and their consumption at the cathode and conversion into gaseous $\mathrm{H}_{2}$. In addition, if not only the characteristics of the anode/solution interface are considered as the source of dynamic instabilities, but the whole electrochemical circuit, many additional external factors may come into play. For instance, if the thermoelectric (Seebeck, Peltier, and Thomson) effects cannot be neglected, they will surely depend on the ambient temperature, the material of electrode clamps, or the height of Al electrodes above the cooled solution, in which they are immersed. Furthermore, if we consider the PAOX pattern as a product of EHD convection in a colloidal system (combining the nano-convective model with the idea of colloidal

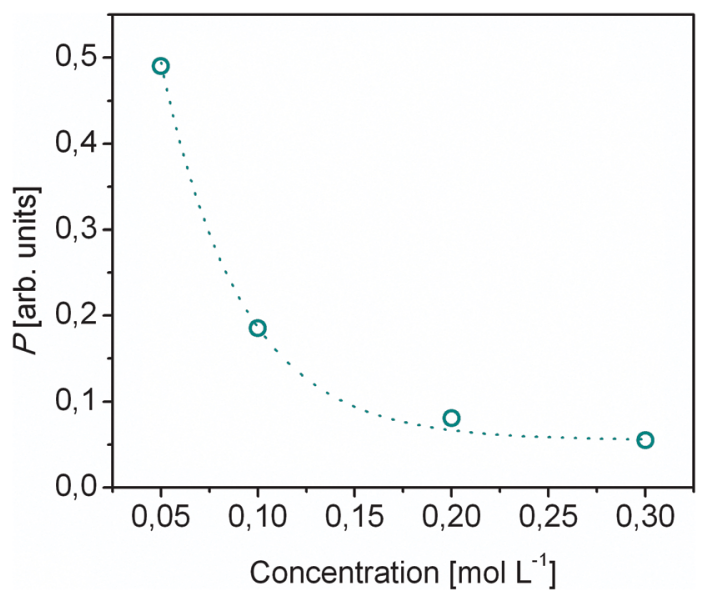

Fig. 5 Dependence of the critical values of criterion $P$ on the oxalic acid anodizing electrolyte concentration, corresponding to moderate (mild) self-ordering voltages. Note that the value of $P$ is equal to 0.055 for $0.3 \mathrm{M}$ oxalic acid electrolyte (on the plot), whereas the average $P$ for three mostly used electrolytes in ref. 20 is equal to 0.057 . gel proposed by Michelson ${ }^{26}$ ), more parameters, such as the mobility of colloidal particles or the thickness of the suspension layer over the anode surface, must be regarded.

The interpolated $P-C$ curve from Fig. 5 can be expressed as:

$$
P=1.47 \mathrm{e}^{-\frac{C}{0.041}}+0.055
$$

where 0.055 is equal to the critical value of $P$ for $0.3 \mathrm{M}$ anodizing electrolyte. Although the physical meaning of the concentrationdependent summand still needs to be clarified, all experimentally observed points obtained for the critical values of the criterion $P$ coincide with the exponential fitting curve with practically no scattering. Thus, there is obviously a strong functional correlation between the critical parameter $P$ for cell pattern appearance and the electrolyte concentration.

\section{Experimental}

Aqueous oxalic acid electrolytes of different concentrations (0.2, 0.1 , and $0.05 \mathrm{~mol} \mathrm{~L}^{-1}$ ) were prepared from oxalic acid dihydrate ( $\geq 99 \mathrm{wt} \%$, extra pure, purchased from Acros Organics) and distilled water $\left(\sigma \approx 2 \mu \mathrm{S} \mathrm{cm}^{-1}\right)$ using standard analytical volumetric flasks. The kinematic viscosities of solutions were measured with Schott Viscoclock Micro-Ubbelohde Viscometer (capillary diameter $0.53 \mathrm{~mm}$ ). Samples' temperature was controlled with Schott CT 1650 thermostat and CK 160 cooler. The densities of solutions at $0{ }^{\circ} \mathrm{C}$ were obtained on an Anton Paar DMA 5000 density meter. The specific conductivity was measured using a WTW LF 538 conductivity meter using a Lauda RM6 thermostat. $\mathrm{pH}$ values were determined using a WTW Digital pH Meter, pH 525. Aluminium sheets (PURALUX $^{\circledR}$, purity 99.5-99.93\%) were anodized in the electrolyte solutions under potentiostatic conditions at $0-0.5{ }^{\circ} \mathrm{C}$ using a Sorensen XEL 250 DC power supply. After the first oxidation for 20 hours, alumina was removed in an etching aqueous solution (0.16 $\mathrm{M} \mathrm{K}_{2} \mathrm{Cr}_{2} \mathrm{O}_{7}$ and $1.5 \mathrm{M} \mathrm{H}_{3} \mathrm{PO}_{4}$ ). Then, the main oxidation step was performed for another 20 hours. The aluminium substrates were used 'as is', without prior electropolishing. Scanning electron microscopy (SEM) studies of the resulting porous films were performed using a Philips XL-30 FEG, operated at 20-25 kV. Samples were mounted on conductive carbon-rich polymer films and sputtered with Pt/Pd alloy. The pore sizes $\left(d_{\mathrm{p}}\right)$ and interpore distances $\left(D_{\text {int }}\right)$ were evaluated using measuring tools of microscope control software or image processing programs. During the statistical treatment, confidence intervals were defined by the significance level $\alpha=0.05$ from 15 random measurements; $d_{\mathrm{p}}$ were measured in high resolution SEM images between the contrasting pore margins (without special visualization techniques), and $D_{\text {int }}$ between centres of randomly chosen neighbouring pores. To bring the confidence intervals in conformity with the electron-scan microscope resolution, the mean $d_{\mathrm{p}}$ and $D_{\text {int }}$ values were approximated to whole numbers.

\section{Conclusions}

As exemplified by our study of aluminium anodization in a series of diluted oxalic acid electrolytes, the formation of a spatial 
hexagonal pattern in porous anodic alumina layers conforms to the same rules as the self-ordering in other electrochemical systems, where the electrohydrodynamic (EHD) convection sets in. The dependence of the critical voltages $\Delta U$ and the critical values of the criterion $P$ on supporting electrolyte concentration can be used for the experimental confirmation of the structureforming role of the EHD convection, in analogy with the earlier studied similar physical and electrochemical cells. In this work, we clarify the nature of possible differences between the theoretically predicted process windows using eqn (1) and the actual experimental observations for some weak or highly diluted electrolytes, which may result from the misconception of the PAOX formation mechanism, and show that the critical value of the criterion $P$ needs to be adjusted individually for every new supporting electrolyte concentration (the optimized values of $P$ for any oxalic acid concentration in the range between $0.05 \mathrm{M}$ and $0.3 \mathrm{M}$ at the temperatures close to $0{ }^{\circ} \mathrm{C}$ can be obtained from the interpolated data in this work). We have demonstrated that the explanation for the hexagonal cell structure in PAOX by the onset of an EHD convective pattern is more viable than the currently prevailing hypothesis suggesting the convective instability of Marangoni type, which is caused by the interfacial tension gradients near the anode during the formation of porous films.

\section{Acknowledgements}

The contribution of Nalan Kalyon and Sharon Jeziorowski to the experimental part of this work is gratefully acknowledged.

\section{References}

1 H. Masuda, in Ordered Porous Nanostructures and Applications, ed. R. B. Wehrspohn, Springer, New York, 2005, vol. 1, pp. 37-55.

2 J. P. Fu, P. Mao and J. Han, Trends Biotechnol., 2008, 26, 311-320.

3 P. W. Bohn, Annu. Rev. Anal. Chem., 2009, 2, 279-296.

4 S. M. Wu, F. Wildhaber, O. Vazquez-Mena, A. Bertsch, J. Brugger and P. Renaud, Nanoscale, 2012, 4, 5718-5723.

5 M. Pashchanka, J. Engstler, J. J. Schneider, V. Siozios, C. Fasel, R. Hauser, I. Kinski, R. Riedel, S. Lauterbach, H. J. Kleebe, S. Flege and W. Ensinger, Eur. J. Inorg. Chem., 2009, 3496-3506.

6 C. B. Gorman, R. J. Petrie and J. Genzer, Macromolecules, 2008, 41, 4856-4865.
7 H. Masuda and K. Fukuda, Science, 1995, 268, 1466-1468.

8 H. Masuda, F. Hasegwa and S. Ono, J. Electrochem. Soc., 1997, 144, L127-L130.

9 A. P. Li, F. Muller, A. Birner, K. Nielsch and U. Gosele, J. Appl. Phys., 1998, 84, 6023-6026.

10 F. Y. Li, L. Zhang and R. M. Metzger, Chem. Mater., 1998, 10, 2470-2480.

11 K. Nielsch, J. Choi, K. Schwirn, R. B. Wehrspohn and U. Gösele, Nano Lett., 2002, 2, 677-680.

12 H. Asoh, K. Nishio, M. Nakao, T. Tamamura and H. Masuda, J. Electrochem. Soc., 2001, 148, B152-B156.

13 Y. Matsui, K. Nishio and H. Masuda, Small, 2006, 2, 522-525.

14 H. Asoh, K. Nishio, M. Nakao, A. Yokoo, T. Tamamura and H. Masuda, J. Vac. Sci. Technol., B, 2001, 19, 569-572.

15 S. Shingubara, K. Morimoto, H. Sakaue and T. Takahagi, Electrochem. Solid-State Lett., 2004, 7, E15-E17.

16 M. A. Kashi and A. Ramazani, J. Phys. D: Appl. Phys., 2005, 38, 2396-2399.

17 A. L. Friedman, D. Brittain and L. Menon, J. Chem. Phys., 2007, 127, 154717.

18 G. D. Sulka and K. G. Parkola, Thin Solid Films, 2006, 515, 338-345.

19 G. D. Sulka and K. G. Parkola, Electrochim. Acta, 2007, 52, 1880-1888.

20 M. Pashchanka and J. J. Schneider, J. Mater. Chem., 2011, 21, 18761-18767.

21 J. Sha, S. J. Lu, Z. S. Su and W. Z. Zhou, Chem. Commun., 2009, 5639-5641.

22 M. Pashchanka and J. J. Schneider, Phys. Chem. Chem. Phys., 2013, 15, 7070-7074.

23 M. Orlik, Self-organization in Electrochemical Systems II, Springer-Verlag, Berlin, Heidelberg, 2012.

24 J. E. Houser and K. R. Hebert, Nat. Mater., 2009, 8, 415-420.

25 G. E. Thompson, Thin Solid Films, 1997, 297, 192-201.

26 C. E. Michelson, J. Electrochem. Soc., 1968, 115, 213-219.

27 M. E. Mata-Zamora and J. M. Saniger, Rev. Mex. Fis., 2005, 51, 502-509.

28 H. Wang and H. W. Wang, Mater. Chem. Phys., 2006, 97, 213-218.

29 B. Baranowski, J. Non-Equilib. Thermodyn., 1980, 5, 67-72.

30 J. W. Diggle, T. C. Downie and C. W. Goulding, J. Electroanal. Chem., 1968, 18, 192-193.

31 J. M. Schneider and P. K. Watson, Phys. Fluids, 1970, 13, 1948-1954. 